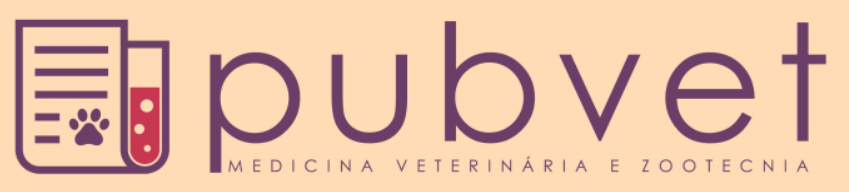

https://doi.org/10.31533/pubvet.v12n8a153.1-7

\title{
Processos tecnológicos empregados no aproveitamento de carcaças suínas e bovinas parcialmente condenadas por cisticercose
}

\author{
Felipe Martins Pastor $^{1 *} \bullet$, Jeniffer Adelaide Adão ${ }^{2}$, Paula Salve Guizardi ${ }^{\bullet}$, Joana \\ Resende Paglis Brunoro ${ }^{4} \bullet$ \\ ${ }^{1}$ Programa de Pós-graduação em Ciências Veterinárias, Universidade Federal do Espírito Santo - UFES - Departamento de Medicina \\ Veterinária/CCAE, Alegre - ES, Brasil.*Autor para correspondência. E-mail: felipempastor@gmail.com \\ ${ }^{2}$ Médica Veterinária, Faculdade de Castelo - Multivix. \\ ${ }^{3}$ Discente do curso de Medicina Veterinária, Faculdade de Castelo - Multivix \\ ${ }^{4}$ Médica Veterinária, Mestre em Ciências Veterinárias pela Universidade Federal de Lavras.
}

RESUMO. A cisticercose é considerada como umas das principais parasitoses que acarretam em prejuízos econômicos na cadeia da carne atualmente. Além disso, a doença se configura como importante zoonose em saúde pública, devido à severidade das implicações que causa no hospedeiro. Com isso, o objetivo deste trabalho foi revisar a bibliografia em busca de processos tecnológicos que permitam um melhor aproveitamento de carcaças suínas e bovinas acometidas pela cisticercose. A salga e o tratamento pelo frio são as principais tecnologias empregadas atualmente no Brasil com o intuito de inviabilizar os cisticercos, em razão da sua eficácia e do melhor custo-benefício para a indústria. A utilização de tais tecnologias é de vital importância para que se obtenha um produto com qualidade e segurança alimentar adequadas para venda ao consumidor final.

Palavras chave: cisticercos, saúde pública, segurança alimentar, zoonose

\section{Technological processes employed in the conditional use of swine and bovine carcasses partially condemned by cysticercosis}

ABSTRACT. Cysticercosis is considered to be one of the main parasitic diseases that cause economic losses in the meat market currently. In addition, the disease is considered an important zoonosis in public health due to the severity of the implications that it causes in the host. Therefore, the objective of this article was to review the literature in search of technological processes that allow a better use of swine and bovine carcasses affected by cysticercosis. The treatment by salting and cold are the main technologies currently used in Brazil to inactivate the parasite, due its effectiveness and the best cost-benefit for the industry. The use of these technologies is essential to obtain a product of satisfactory quality and safety for sale to the final consumer.

Keywords: cysticercus, food safety, public health, zoonosis

\section{Procesos tecnológicos empleados en el aprovechamiento de canales porcinas y bovinas parcialmente condenadas por cisticercosis}

RESUMEN. La cisticercosis es considerada como una de las principales parasitosis que causa perjuicios económicos en la cadena de la carne actualmente. Además, la enfermedad se configura como importante zoonosis en salud pública, debido a la severidad de las implicaciones que causa en el huésped. Con ello, el objetivo de este trabajo fue revisar la bibliografía en busca de procesos tecnológicos que permitan un mejor aprovechamiento de carcasas 'porcinas y bovinas acometidas por la cisticercosis. La salazón y el tratamiento 
por el frío son las principales tecnologías empleadas actualmente en Brasil con el propósito de inviabilizar los cisticercos, en razón de su eficacia y del mejor costo-beneficio para la industria. La utilización de estas tecnologías es de vital importancia para que se obtenga un producto con calidad y seguridad alimentarias adecuadas para la venta al consumidor final.

Palabras clave: cisticercos, salud pública, seguridad alimentaria, zoonosis

\section{Introdução}

A cisticercose é uma das doenças parasitárias de maior importância no cenário econômico do mercado de carnes, causando diversos prejuízos em relação à alta taxa de condenação de carcaças dos animais acometidos. Além disso, também representa um grande risco à saúde pública, por se tratar de uma zoonose com implicações severas, como lesões teciduais e danos neurológicos. Dependendo do grau de infestação que a carcaça apresentar durante a inspeção post-moterm, esta pode seguir diversos destinos tecnológicos visando o melhor aproveitamento, evitando assim o descarte desnecessário de grandes quantidades de carne.

O objetivo deste trabalho foi revisar a bibliografia em busca de processos tecnológicos que permitam um aproveitamento otimizado de carcaças suínas e bovinas acometidas pela cisticercose.

\section{Revisão de literatura}

\section{Aspectos econômicos e biológicos da cisticercose}

Os alimentos, em especial aqueles de origem animal, ao mesmo tempo em que se constituem como principal mercadoria entre os países importadores e exportadores do mundo, são um dos meios de maior importância na disseminação de doenças infecciosas transmissíveis por alimentos (DTAs). A segurança sanitária dos alimentos é uma questão de saúde pública, sendo um desafio que demanda colaboração internacional a fim de garantir a proteção do alimento, bem como do consumidor final (Figueiredo \& Miranda, 2011). Dentre as principais DTAs, o Complexo TeníaseCisticercose tem atenção especial dentro da inspeção de produtos de origem animal, por se tratar de uma zoonose e repercutir negativamente no mercado da carne (Rezende et al., 2006).

Khaniki et al. (2010) e Pereira et al. (2006) avaliam que anualmente há prejuízos de cerca de USD 410.000 até 420.000 .000 com carcaças condenadas pela cisticercose bovina. No cenário nacional, Guimarães-Peixoto et al. (2012) estimam que no período de 2004 a 2008, a cisticercose levou à perdas econômicas da ordem de aproximadamente R $\$ 120$ milhões no Estado do Paraná.

Os cisticercos, que são larvas das tênias, se alojam em diferentes tecidos de seu hospedeiro intermediário, e quando viáveis, apresentam-se sob a forma de vesículas transparentes contendo um escólex (Prata \& Oliveira, 2011). Quando o hospedeiro intermediário é o bovino, trata-se de Cysticercus bovis, que origina a Taenia saginata em sua forma adulta; nos casos onde o hospedeiro intermediário é o suíno, tem se $C$. cellulosae, e Taenia sollium como sua respectiva forma adulta (Pardi et al., 2001; Prata \& Oliveira, 2011).

As formas adultas de Taenia são parasitas intestinais do ser humano, porém, de acordo com Abreu et al. (2001) C. cellulosae demanda maior precaução, pois diferente de C. bovis, este também acomete os seres humanos como hospedeiros intermediários, com repercussões ainda mais severas ao atingir os tecidos da região ocular, do cérebro e musculatura, causando cegueira, sinais neurológicos e miosites.

No hospedeiro intermediário, o cisticerco se aloja com maior frequência em órgãos que possuem maior irrigação sanguínea, como coração, músculos mastigadores e língua (Fischmann, 1994; Takayanagui, 1996). Locais como o diafragma, os músculos dos membros e, ocasionalmente, a gordura, os pulmões, o fígado, linfonodos e rins também podem ser acometidos (Santos et al., 2001).

Determinados fatores como religião, cultura e condição econômica tendem a deixar indivíduos susceptíveis a contrair a teníase através da ingestão do cisticerco na carne crua ou mal cozida. Dias et al. (1991) descreve que, em um grupo de 2500 pessoas acometidas pelo parasito adulto, $90 \%$ delas relataram ter ingerido carne crua conscientemente. Como questão cultural, alguns pratos típicos como carpaccio, bife tártaro e o quibe cru são preparados com carne crua ou mal cozida (Pfuetzenreiter \& Ávila-Pires, 2000; Germano \& Germano, 2015).

Como medidas preventivas para todas as formas de teníase-cisticercose, podem ser citadas 
o saneamento básico, higiene alimentar, cozimento correto de carnes de suínos e bovinos, tratamento de pessoas e animais infectados, além da inspeção post-mortem de animais destinados a consumo humano (Valadares, 1997). Nesse contexto, uma inspeção criteriosa das carcaças nos frigoríficos é uma das formas mais eficazes no controle do complexo Teníase-Cisticercose (Ungar \& Germano, 1992).

\section{Decisões tomadas durante a inspeção post- mortem}

O Art. 176 do RIISPOA define que na inspeção de rotina, determinados órgão devem ser priorizados na procura pelos cisticercos, como a cabeça, língua e coração. Na cabeça, se observam e incisam os masseteres e pterigoideos internos e externos; na língua, se observa, palpa e incisa longitudinalmente. No coração, após observação, realiza-se incisão longitudinal da base à ponta, através da parede do ventrículo esquerdo e do septo interventricular, e se praticam incisões largas nas paredes dos ventrículos; caso já se tenha notado a presença do cisticerco em algum dos órgãos anteriores, fazem-se mais incisões na musculatura do coração, a fim de garantir uma inspeção detalhada (Brasil, 1997).

Quando se encontram infestações intensas, nas quais há mais de um cisto em incisões em uma região correspondente à palma da mão, a carcaça deve ser totalmente condenada e destinada à graxaria. O art. 197 do RIISPOA permite que nesse caso, apenas o tecido adiposo dos suínos infestado por C. cellulosae seja utilizado para fabricação de banha, devendo-se rejeitar o restante da carcaça (Brasil, 2017).

A condenação parcial da carcaça acometida pela cisticercose se dá nas situações em que, por exemplo, for encontrado um único cisto calcificado, que após ser removido juntamente com o tecido circundante, pode ser liberada a carcaça. Naqueles casos onde há infestação discreta ou moderada, todos os cistos e tecidos circunvizinhos acometidos são removidos, e então o restante segue para o tratamento pelo frio ou pela salmoura (Brasil, 2017).

\section{Processos tecnológicos utilizados na inviabilização dos cisticercos}

Dentro da indústria, existem diversos processos aos quais a carcaça acometida por cisticercose pode ser submetida, como tratamento pelo calor, frio, salga, radiação, entre outros.

\section{Calor}

A utilização de calor para o aproveitamento condicional de carcaças está veiculada ás instalações do estabelecimento, já que este deve possuir dependências específicas para tal aproveitamento. Apesar de não ser um método comumente utilizado para o aproveitamento condicional de carnes no Brasil, o calor é um método bastante eficaz, já que os cisticercos não resistem a temperaturas superiores a $50^{\circ} \mathrm{C}$. No entanto, deve-se considerar que não é fácil aquecer o centro de uma massa de carne a esta temperatura: presuntos cozidos chegam, no máximo, a $46^{\circ} \mathrm{C}$ em duas horas, e em carnes defumadas a quente, o cisticerco tem grandes chances de sair ileso da ação do calor, já que a temperatura preconizada varia de 50 a $120^{\circ} \mathrm{C}$ (Rey, 2001).

De acordo com Brasil (2017), o tratamento pelo calor que garanta a inativação de patógenos pode ser realizado na indústria de três formas distintas: cozimento a no mínimo $76,6^{\circ} \mathrm{C}$ por 30 minutos; fusão pelo calor a $121^{\circ} \mathrm{C}$ ou temperatura superior a esta; ou esterilização pelo calor úmido seguida de resfriamento rápido.

\section{$\underline{\text { Frio }}$}

De acordo com o artigo 185 do RIISPOA, carcaças com infecção leve, com um cisto viável, podem ser tratadas pelo frio (Brasil, 2017). Quando se utiliza desta técnica, as carcaças são dispostas em câmaras onde a temperatura deve atingir marcas não superiores a $10^{\circ} \mathrm{C}$ negativos. Ali, as carcaças devem permanecer por um período mínimo de 10 dias, a fim de garantir a inativação de cisticercos que ainda possam ter passado despercebidos durante a inspeção (Brasil, 2014). Segundo Roça (2011a), o método utilizado é o de congelamento em corrente de ar, empregando-se salas equipadas com ventiladores, que emitem correntes de ar com velocidade de 5 a $6 \mathrm{~m} / \mathrm{s}$.

Quando a carne é submetida á temperaturas superiores a $0^{\circ} \mathrm{C}$, não há interferência na sobrevivência dos cisticercos. Sotelo et al. (1986) e Benenson (1992) afirmam que a inativação dos cisticercos é eficaz quando a 
carne é mantida a $-5^{\circ} \mathrm{C}$ por 4 dias, ou por 3 dias a $-15^{\circ} \mathrm{C}$. Rey $(2001)$ concluiu que temperaturas inferiores a $-15^{\circ} \mathrm{C}$ são eficazes na eliminação dos cisticercos em até 6 dias.

\section{$\underline{\text { Salga }}$}

O tratamento das carcaças através da salga é uma das medidas utilizadas pelo Serviço de Inspeção Sanitária quando são encontradas infecções discretas ou moderadas (Almeida et al., 2008).

A Instrução Normativa $N^{\circ} 007$ de 30/08/2016, do Instituto de Defesa Agropecuária e Florestal do Espírito Santo (IDAF, 2016), prevê que a salga seja utilizada como método de tratamento em carcaças acometidas pela cisticercose: devem ser utilizados cortes de $3,5 \mathrm{~cm}$ de espessura, no máximo, e dispostos em salmoura com no mínimo 24 graus Baumé. O período que os cortes devem permanecer no tratamento é de no mínimo 21 dias, para que haja ação suficiente do sal na inativação dos cisticercos, sendo que esse tempo pode ser reduzido para 10 dias quando a carne for mantida a temperatura de $1^{\circ} \mathrm{C}$ ou menor que esta (Brasil, 2017).

Bartels (1971) relata que em uma salmoura a $25 \%$, todos os cisticercos da carne suína são destruídos em até 14 dias, enquanto Thornton (1969) descreve que o processo leva de 3 a 4 semanas em pedaços de carne de até $2,5 \mathrm{~kg}$. Quando se utiliza uma salmoura com a concentração de $50 \mathrm{~g}$ de sal por kg de carne, em 2 a 3 semanas já se tem o cisticerco inviabilizado (Pessôa \& Martins, 1982).

De acordo com Riccetti (1975) a salga seca inativa eficazmente os Cytiscercus cellulosae em 24 horas, caso estes estejam localizados superficialmente. Para cisticercos mais profundos, a inativação ocorre no mesmo período, desde que a fatia de carne não contenha mais do que $3 \mathrm{~cm}$. Riccetti et al. (1977) comprovou que fatias de 6 $\mathrm{cm}$ de espessura sofrem inativação dos cisticercos em 7 dias, em temperatura ambiente. $\mathrm{O}$ autor também descreve que quando se associa a temperatura de $4^{\circ} \mathrm{C}$ ao processo de salga, este deve durar no mínimo 15 dias.

Almeida (2008) relata que em 16 horas é possível inativar os cisticercos localizados superficialmente em pedaços de carne bovina de 2 $\mathrm{cm}$ de espessura, quando se utiliza solução salina a 7\%. Braga et al. (2006) conclui que a solução salina de 7\% também é capaz de inativar completamente os cisticercos superficiais em 24 horas de salmoura. Com esse mesmo período, Carrijo et al. (2005) descrevem que a inviabilização do cisticerco em solução salina 7\% pode ser feita em pedaços de carne de $5 \mathrm{~cm}$ de comprimento por $3 \mathrm{~cm}$ de espessura.

Para Almeida et al. (2008), esses resultados mostram que a inviabilização do cisticerco em períodos curtos, como de 24 horas, é relacionado à localização da larva, sendo que este tempo não é suficiente para eliminar os cistos mais profundos. Para esse tipo de larva, Ricetti (1975) recomenda um período de tempo de 96 horas para inviabilização total dos cisticercos.

Em relação à bioquímica da inviabilização do cisticerco pela salga, Roça (2011b) relata que as altas concentrações de sal elevam a pressão osmótica da carne, fazendo com que as proteínas se precipitem e reduza a retenção de água. Com as proteínas desnaturadas e baixa quantidade de água disponível, o cisticerco não consegue sobreviver nesse meio. Além disso, concentrações acima de $13 \%$ possuem a ação de rompimento da membrana do cisticerco, levando a morte da larva (Gouvêa \& Gouvêa, 2007).

Quanto aos equipamentos necessários ao processo, a portaria $\mathrm{n}^{\circ} 914$, de 12 de setembro de 2014 (Brasil, 2014), estabelece que o frigorífico deva possuir instalações exclusivas para a salga, cura e embalagem desses produtos. Todos os instrumentos e equipamentos utilizados para a salga, como os tanques, mesas e tombadores, deverão ser fabricados em material liso, sem ranhuras e devem ser resistentes à corrosão. A mesma portaria ainda prevê que as pilhas de carne salgada devem ser identificadas com a data de produção, e não devem ser dispostas diretamente sobre o piso, não devendo estar encostadas em paredes e colunas.

O processo tecnológico da salga das carnes compreende diversas etapas, e pode ser mais bem compreendido no Fluxograma 1. Finalizados estes processos, as carnes acometidas pela cisticercose podem ser consideradas seguras para consumo, e seguem outros destinos tecnológicos de acordo com o objetivo do frigorifico e a demanda do mercado. 


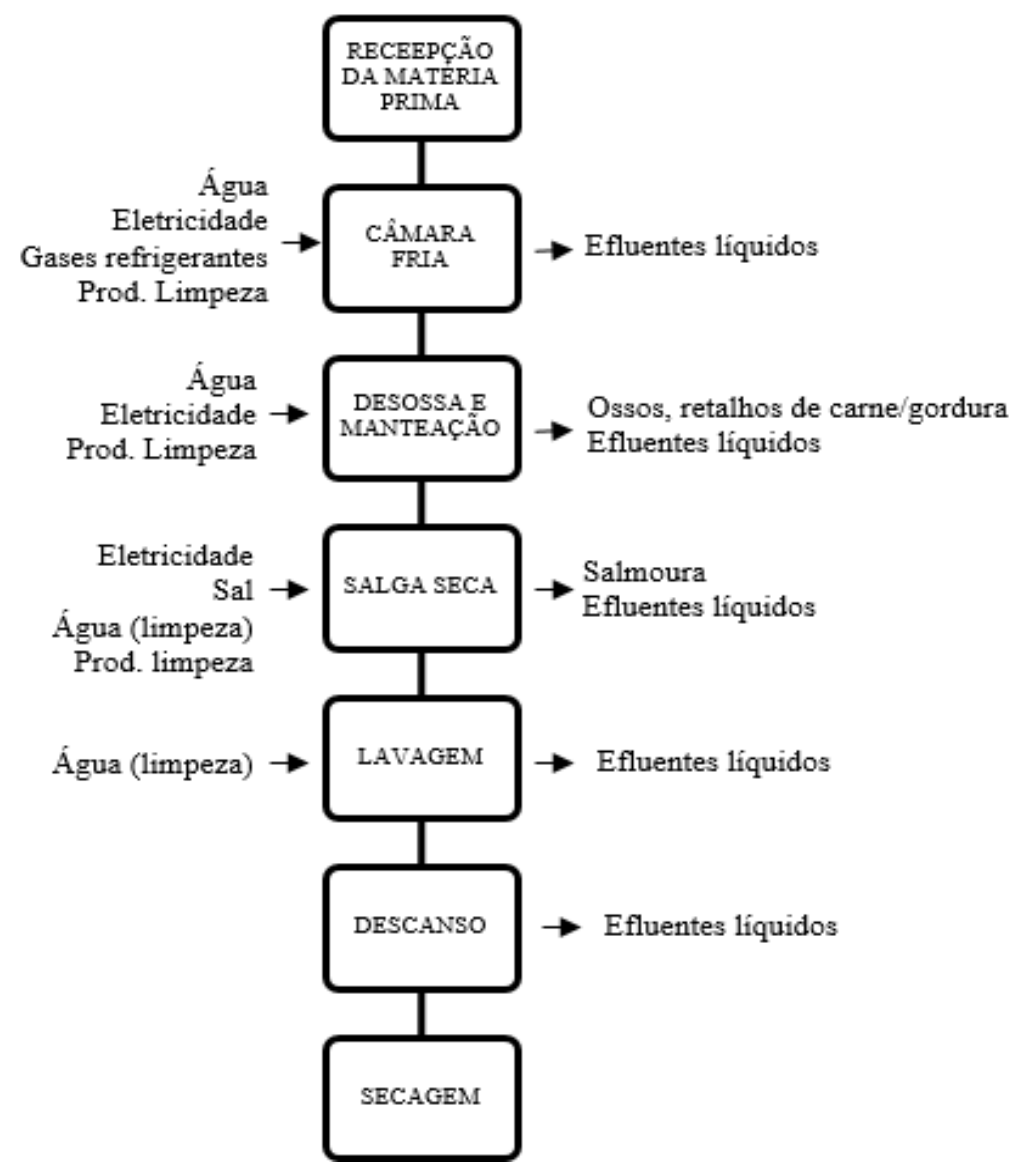

Fluxograma 1. processo de salga de carnes. Fonte: adaptado de Pacheco (2006).

\section{Considerações finais}

Embora o complexo teníase-cisticercose seja um problema em saúde pública, há medidas a serem tomadas que inviabilizem os cisticercos presentes em carnes bovina e suína infectadas. E dentro destas medidas, a inspeção sanitária assume importante papel, onde uma vez detectados estes cistos, a carne será encaminhada para diferentes processos tecnológicos, definidos de acordo com o grau de infecção das carnes.

Dentre os processos tecnológicos utilizados como medida de inativação dos cistos encontramse a salga e o congelamento, sendo estes os métodos mais utilizados no Brasil, devido ao melhor custo benefício para indústria e relativa eficácia na inativação do cisticerco. A utilização de tais tecnologias é de vital importância para que se obtenha um produto com qualidade e segurança adequadas prra a venda ao consumidor final.

\section{Referências}

Abreu, R. L. de, Sousa, M. R. P. de, Correia, P, G., Mathias, S. P., Leite, F. 2001. Ocorrência de Cisticercos SP em carnes comercializadas no município de Seropédica - RJ. Revista Higiene Alimentar, 15, (84), 21-23.

Almeida, L. P., Carrijo, K. F., Reis, D. O. \& Moreira, M. D. 2008. Eficácia de solução salina 7\% na inviabilização de Cysticercus bovis em postas de carne bovina em período de tempo de 4 e 16 horas à temperatura ambiente. Veterinária Notícias, 14(1), 37-40.

Aquino, C. A. M. Viabilidade de cisticercos em diferentes concentrações de sal. 2003. Faculdade de Medicina Veterinária, Universidade Federal de Uberlândia, Uberlândia-MG, 16.

Bartels, H. 1971. Inspección veterinaria de la carne (491 p.). Acribia, Zaragoza, ES.

Benenson, A. S. 1992. El control de las enfermedades transmisibles en el hombre $\left(15^{\mathrm{a}}\right.$ ed., 618 p.). OPS/OMS, Washington, US.

Braga, J. S., Borges, T. D., Almeida, L. P. \& Moreira, M. D. 2006. Eficácia da solução salina $7 \%$ na inviabilização de cisticercos. Veterinária Notícias, 12(2), 72.

Brasil, Ministério da Agricultura, Pecuária e Abastecimento. 2014. Portaria $\mathrm{N}^{\circ}$ 914, de 12 de Setembro de 2014. DOU de 15/09/2014 $\left(\mathrm{n}^{\circ}\right.$ 
177, Seção 1, pág. 4). Disponível em: http://www.lex.com.br/legis_25956053_POR TARIA_N_914_DE_12_DE_SETEMBRO_D E_2014.aspx. Acesso em: 29 de março, 2017.

Brasil, Ministério da Agricultura, Pecuária e Abastecimento. 2017. RIISPOA Regulamento de Inspeção Industrial e Sanitária de Produtos de Origem Animal. Decreto $n^{\circ}$ 9.013, de 29 de março de 2017.

Brasil, Ministério da Agricultura. 1997. Regulamento da inspeção Industrial e Sanitária de Produtos de Origem Animal (RISPOA). Brasília, 1950. 165p. (aprovado pelo decreto ${ }^{\circ}$ 30.691 de 29.03.52, alterado pelo decreto $\mathrm{n}^{\circ}$ 2.244 de 04.06.1997).

Carrijo, K. F., AlmeidA, L. P. \& Moreira, M. D. 2005. Inviabilização de cisticercos: um estudo utilizando solução salina 7\% por um período de 24 horas. Revista Higiene Alimentar, 19(136), 109-111.

Dias, R. M. D. S., Silva, M. I. P. G., Mangini, A. C. S., Vellosa, S. A. G., Torres, D. M. A. G. V., Silva, R. M. \& Vas, A. J. 1991. Ocorrência de Taenia $s p$ na população atendida no Laboratório Central do Instituto Adolfo Lutz, São Paulo, SP, Brasil. (1960/1989). Revista do Instituto de Medicina Tropical de São Paulo, 33, (2), 147-151.

IDAF, Instituto de Defesa Agropecuária e Florestal do Espírito Santo. 2016. Instrução Normativa $N^{\circ} 007$ de 30/08/2016. Instituto de Defesa Agropecuária e Florestal do Espírito Santo. Publicado no DOE em 31 de agosto de 2016.

Figueiredo, A. V. A. \& Miranda, M. S. 2011. Análise de risco aplicada aos alimentos no Brasil: perspectivas e desafios. Ciência e Saúde Coletiva, 16(4), 2251-2262.

Fischmann, A. 1994. Vigilância epidemiológica. In: Rouquayrol, M. Z. Epidemiologia e Saúde (4a ed., p. 421-441). MEDSI, Rio de Janeiro, Brasil.

Germano, L. M. P. \& Germano, S. I. M. 2015. Higiene e vigilância Sanitária de alimentos: 5ed. São Paulo, Manole. Cap.21 p. 463 - 477.

Gouvêa, J. A. G. \& Gouvêa, A. A. L. 2007. Tecnologia de fabricação da carne de sol. Dossiê Técnico. Rede de Tecnologia da Bahia - RETEC/BA. Disponível em: http://www.ebah.com.br/content/ABAAAewx 4AL/dossie-tecnico-tecnologia-fabricacaocarne-sol. Acesso em: 01 de abril, 2017.
Guimarães-Peixoto, R. P. M., Souza, V. K., Pinto, P. S. A. \& Santos, T. O. 2012. Distribuição e identificação das regiões de risco para a cisticercose bovina no Estado do Paraná. Pesquisa Veterinária Brasileira, 32(10), 975979.

Khaniki, G. H. R. J., Raei, M., Kia, E. B., Motevalli, A. H. \& Selseleh, M. 2010. Prevalence of bovine cysticercosis in slaughtered cattle in Iran. Tropical Animal Health and Production, 42(3),141-143.

Pacheco, J. W. 2006. Guia técnico ambiental de frigoríficos - industrialização de carnes (bovina e suina) (85 p.). CETESB, São Paulo, BR.

Pardi, M. C., Santos, I. F., Souza, E. R. \& Pardi, H. S. 2001. Ciência, Higiene e Tecnologia da Carne ( $2^{\mathrm{a}}$ ed., v. 2, p. 524). UFG, Goiânia, Brasil.

Pereira, M. A. V., Schwanz, V. S. \& Barbosa, C. G. 2006. Prevalência da cisticercose em carcaças de bovinos abatidos em matadourosfrigoríficos do Estado do Rio de Janeiro, submetidos ao controle do serviço de inspeção federal (SIF-RJ), no período de 1997 a 2003. Arquivos do Instituto Biológico, 73(1), 83-87.

Pessôa, S. B. \& Martins, A. V. 1982. Parasitologia Médica (11a ed., 872 p.). Guanabara Koogan, Rio de Janeiro, BR.

Pfuetzenreiter, M. R. \& Ávila-pires, F. D. 2000. Epidemiologia da Teníase/Cisticercose por Taenia solium e Taenia saginata. Ciência Rural, 30(3), 541-548.

Prata, L. F. \& Oliveira, L. G. 2011. Inspeção e tecnologia da carne (p. 163). Funep, Jaboticabal, BR.

Rey, L. 2001. Parasitologia - parasitos e doenças parasitárias do homem nas Américas e na África (3a ed., 731 p.). Guanabara Koogan, Rio de Janeiro, BR.

Rezende R.B.C., Fernadez A.T., Costa F. \& Silva T.J.P. 2006. Ocorrência de cisticercose em bovinos abatidos clandestinamente no município de Silva Jardim, RJ. Revista Higiene Alimentar, 21:103-109 p.

Riccetti, R. V. 1975. Estudo sobre a atuação do cloreto de sódio na inativação do Cisticercus cellulosae em massas musculares de suínos naturalmente infectados. Revista $d a$ Faculdade de Medicina Veterinária e Zootecnia de Universidade de São Paulo, 12, 259-68. 
Riccetti, R. V., Panetta, J. C. \& Barbuto, O. J. M. 1977. Efeito da temperatura e do cloreto de sódio (salga seca) sobre a viabilidade do Cysticercus cellulosae em segmentos de carne suína de diferentes espessuras. Revista da Faculdade de Medicina Veterinária e Zootecnia de Universidade de São Paulo, 14(1), 113-122.

Roça, R. O. 2011a. Congelação. FAC/Unesp, Botucatu, BR. Disponível em http://www.fca.unesp.br/Home/Instituicao/De partamentos/Gestaoetecnologia/Teses/Roca10 9.pdf. Acesso em 25 mar 2017.

Roça, R. O. 2011b. Cura de carnes. FCA/Unesp, Botucatu, BR. Disponível em http://www.fca.unesp.br/Home/Instituicao/De partamentos/Gestaoetecnologia/Teses/Roca11 1.pdf. Acesso em 25 mar 2017.

Santos, I. F., Mano, S. B., Tortelly, R., Santos, M. L. S. \& Silva, D. A. S. 2001. Estudo da localização do Cysticercus bovis em corações de bovinos abatidos sob inspeção. Revista Higiene Alimentar, 15(89), 41-43.
Sotelo, J., Rosas, N. \& Palencia, G. 1986. Freezing of infested pork muscle kills cysticerci. JAMA, 256:893-894.

Takayanagui, O. M. 1996. Notificação compulsória da cisticercose em Ribeirão Preto - SP. Arquivos de Neuro-Psiquiatria, 54(4), 557-564.

Thornton, H. 1969 Compêndio de inspeção de carnes (665 p.). FARMOP, Rio de Janeiro, Brasil.

Ungar, M. L. \& Germano, P. M. L. 1992. Prevalência da cisticercose bovina no Estado de São Paulo (Brasil). Revista de Saúde Pública, 26, (3), 167-172.

Valadares, M. D. M. 1997. Complexo teníase/cisticercose. Revista Brasileira de Parasitologia Veterinária, 6, 1-62.

Recebido: 16 Jul. 2018

Aprovado: 7 Jul. 2018

Publicado: 6 Ago. 2018

Licenciamento: Este artigo é publicado na modalidade Acesso Aberto sob a licença Creative Commons Atribuição 4.0 (CC-BY 4.0), a qual permite uso irrestrito, distribuição, reprodução em qualquer meio, desde que o autor e a fonte sejam devidamente creditados. 